\title{
摩尔比法测定磺基水杨酸铜组成与稳定常数的研究
}

彭敏, 石建新 ${ }^{*}$, 王周, 李莲云

中山大学化学学院, 广州 510275

摘要: 通过实验探讨摩尔比法测定磺基水杨酸铜配合物组成与稳定常数的可行性。实验测得磺基水杨酸铜在 $\mathrm{pH}$ 为 5 时络 合比为 1 , 绝对稳定常数 $\lg K=9.63$, 与理论值 9.52 接近, 表明本文设计的摩尔比法测定磺基水杨酸铜组成与稳定常数的 实验方案可行。

关键词: 摩尔比法; 磺基水杨酸铜; 稳定常数

中图分类号: G64; O6-39

\section{Study about the Composition and Stability Constant of Sulfosalicylic Acid-Copper(II) Complex by Mole Ratio Method}

\author{
Min Peng, Jianxin Shi ${ }^{*}$, Zhou Wang, Lianyun Li \\ School of Chemistry, Sun Yat-Sen University, Guangzhou 510275, China.
}

\begin{abstract}
The feasibility of using the mole-ratio method for study of the formation and stability constant of sulfosalicylic acid-copper(II) complex was explored. The experimental result revealed that the chelate-to-copper ratio was $1: 1$ at $\mathrm{pH}=5$, and its absolute stability constant was found to be $\lg K=9.63$, which was close to the value 9.52 from the handbook.
\end{abstract}

Key Words: Mole ratio method; Sulfosalicylic acid-copper(II) complex; Stability constant

传统的配合物稳定常数测定方法 ${ }^{[1]}$ 有 $\mathrm{pH}$ 电位法、分光光度法、电导滴定法、量热滴定法、极谱 法、离子交换法、相平衡法等。基础无机实验—磺基水杨酸铜配合物组成与稳定常数的测定使用 的是分光光度法。分光光度法分为等物质的量系列法和物质的量比法 ${ }^{[2]}$ (又称摩尔比法、饱和法, 以 下统称摩尔比法), 实验一般是采用等物质的量系列法进行。检索有关摩尔比法的中文文献, 有高吉 刚等 ${ }^{[3]}$ 研究 $\mathrm{Cu}$ 和4-(5-氯-2-吡啶偶氮)-1,2-二氨基苯(5-Cl-PADAB)体系的稳定常数, 张淑艳等 ${ }^{[4]}$ 研究 $N-$ 苯甲酰基- $N^{\prime}, N^{\prime}$-二苯代硫脲合铜(II)配合物组成, 但未见有摩尔比法测定磺基水杨酸铜稳定常数的相 关文献。本文通过实验研究摩尔比法测定磺基水杨酸铜组成与稳定常数的可行性, 并对实验结果进 行探讨。

\section{1 实验部分}

\section{1 实验试剂与主要仪器}

S22PC分光光度计电子分析天平(梅特勒-托利多EL204), 雷磁pHs-3C酸度计, 电子秤(梅特勒-托

收稿: 2020-09-28; 录用: 2020-11-12; 网络发表: 2020-12-22 
利多PL602-E), 电磁摚拌器(IKA C-MAG HS7)。

$\mathrm{NaOH}\left(1.0 \mathrm{~mol} \cdot \mathrm{L}^{-1}, 0.05 \mathrm{~mol} \cdot \mathrm{L}^{-1}\right), \mathrm{HNO}_{3}\left(0.05 \mathrm{~mol} \cdot \mathrm{L}^{-1}\right)$ 。

$0.05000 \mathrm{~mol} \cdot \mathrm{L}^{-1}$ 硝酸铜溶液的配制: 准确称取 $12.078 \mathrm{~g}$ 三水合硝酸铜用水溶解, 转移至 $1000 \mathrm{~mL}$ 容量瓶中并用水定容。

$0.10 \mathrm{~mol} \cdot \mathrm{L}^{-1}$ 硝酸钾溶液的配制: $10.0 \mathrm{~g}$ 硝酸钾溶于 $1 \mathrm{~L}$ 水中。

$0.2500 \mathrm{~mol} \cdot \mathrm{L}^{-1} 5$-磺基水杨酸(简写为 $\mathrm{H}_{3} \mathrm{~L}$ )溶液的配制: 准确称取 $15.888 \mathrm{~g}$ 二水合 5 -磺基水杨酸用 水溶解, 转移至 $250 \mathrm{~mL}$ 容量瓶中并用水定容。

$\mathrm{pH}$ 计校正用标准缓冲液 $(4.0,6.86)$ : 由厂家提供的雷磁 $\mathrm{pH}$ 缓冲剂粉包按要求配制。

所用试剂均为分析纯, 实验用水为去离子水。

\section{2 实验方案}

1) 用 $0.05000 \mathrm{~mol} \cdot \mathrm{L}^{-1}$ 硝酸铜溶液和 $0.2500 \mathrm{~mol} \cdot \mathrm{L}^{-1} \mathrm{H}_{3} \mathrm{~L}$, 在 12 个 $50 \mathrm{~mL}$ 烧杯中按表 1 的要求配制系 列混合溶液(硝酸铜溶液由 $10 \mathrm{~mL}$ 移液管移取; 1-7号溶液所需 $\mathrm{H}_{3} \mathrm{~L}$ 采用 $2 \mathrm{~mL}$ 吸量管移取, $8-12$ 号的 $\mathrm{H}_{3} \mathrm{~L}$ 由 $25 \mathrm{~mL}$ 酸式滴定管放液取得)。

2) 在 $\mathrm{pH}$ 计及电磁搅拌器辅助下调节系列溶液 $\mathrm{pH}$ 为 4.70 。

3) 调好 $\mathrm{pH}$ 的溶液分别定量转移到 $50 \mathrm{~mL}$ 容量瓶, 并用已调好 $\mathrm{pH}$ 为 4.70 的 $0.1 \mathrm{~mol} \cdot \mathrm{L}^{-1}$ 硝酸钾定容。

4) 在 S22PC分光光度计上选波长为 $440 \mathrm{~nm}$, 用 $1 \mathrm{~cm}$ 比色典, 以 $0.1 \mathrm{~mol} \cdot \mathrm{L}^{-1}$ 硝酸钾作参比, 分别 测定各溶液的吸光度, 结果见表1。

表1 摩尔比法系列混合溶液的配制及其吸光度测定

\begin{tabular}{ccccc|ccccc}
\hline 溶液编号 & $V_{\mathrm{H}_{3} \mathrm{~L}} / \mathrm{mL}$ & $V_{\mathrm{Cu}\left(\mathrm{NO}_{3}\right)_{2}} / \mathrm{mL}$ & $n=\frac{c_{\mathrm{L}}}{c_{\mathrm{M}}}$ & 吸光度 $A$ & 溶液编号 & $V_{\mathrm{H}_{3} \mathrm{~L}} / \mathrm{mL}$ & $V_{\mathrm{Cu}\left(\mathrm{NO}_{3}\right)_{2}} / \mathrm{mL}$ & $n=\frac{c_{\mathrm{L}}}{c_{\mathrm{M}}}$ & 吸光度 $A$ \\
\hline 1 & 0 & 10 & 0 & 0.003 & 7 & 2.0 & 10 & 1.00 & 0.435 \\
2 & 0.2 & 10 & 0.10 & 0.055 & 8 & 4.0 & 10 & 2.00 & 0.546 \\
3 & 0.4 & 10 & 0.20 & 0.113 & 9 & 6.0 & 10 & 3.00 & 0.579 \\
4 & 0.6 & 10 & 0.30 & 0.163 & 10 & 8.0 & 10 & 4.00 & 0.603 \\
5 & 1.0 & 10 & 0.50 & 0.258 & 11 & 10 & 10 & 5.00 & 0.606 \\
6 & 1.5 & 10 & 0.75 & 0.364 & 12 & 12 & 10 & 6.00 & 0.608 \\
\hline
\end{tabular}

\section{2 结果与讨论}

\section{1 摩尔比法作图确定磺基水杨酸铜的络合比}

由表1所得数据作图, 如图1所示。

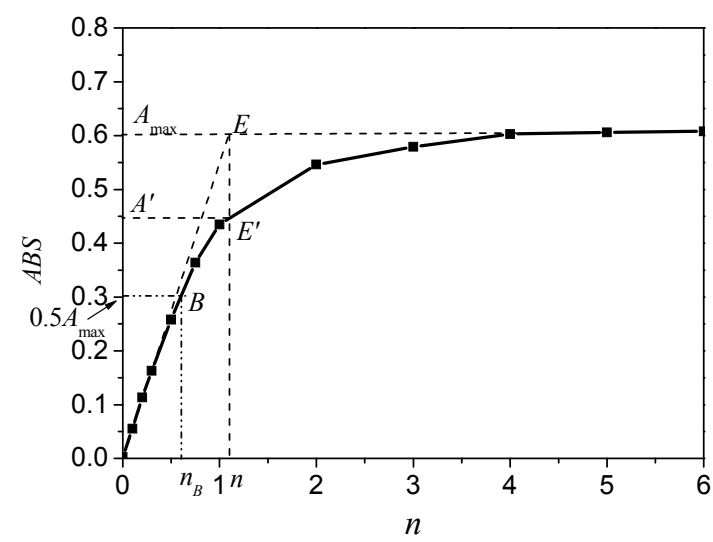

图1 摩尔比法测定磺基水杨酸铜的组成和稳定常数 
分析表 1 数据可知, 当 $n \geq 4$ 时, 溶液的吸光度基本保持不变, 当 $n \leq 0.3$ 时, 随着 $n$ 值增大, 溶液 吸光度也线性增大; 根据摩尔比法原理作图 ${ }^{[2]}$, 以图 1前四个点、后三个点分别作直线, 两者交于 $E$ 点, $E$ 点对应的横坐标 $n$ 即为配合物的络合比, 其值见表 2 ; 对应纵坐标 $A_{\max }$ 为磺基水杨酸铜吸光度 最大值, 近似等于配合物未发生解离时的吸光度。

表2 由图1所得实验数据汇总

\begin{tabular}{cccccc}
\hline$A^{\prime}$ & $A_{\max }$ & $0.5 A_{\max }$ & $n_{B}$ & $0.5 A_{\max }$ 对应 $c_{\mathrm{L}} /\left(\mathrm{mol} \cdot \mathrm{L}^{-1}\right)$ & $\alpha$ \\
\hline 0.447 & 0.606 & 0.303 & 0.608 & 0.00608 & 0.262 \\
\hline $0.5 A_{\max }$ 对应 $\mathrm{H}_{3} \mathrm{~L}$ 浓度 $c_{\mathrm{L}}=n_{B} \cdot c_{\mathrm{M}}, c_{\mathrm{M}}$ 为溶液中铜离子的总浓度, 其值为 $0.01000 \mathrm{~mol} \cdot \mathrm{L}^{-1} ;$ 解离度 $\alpha=\frac{A_{\max }-A^{\prime}}{A_{\max }}$
\end{tabular}

\section{2 磺基水杨酸铜稳定常数的计算}

对于配合物 $\mathrm{ML}_{n}$, 在溶液中有如下关系:

$$
\mathrm{M}+n \mathrm{~L} \rightleftharpoons \mathrm{ML}_{n}
$$

稳定常数表示为:

$$
K_{\text {稳 }}=\frac{\left[\mathrm{ML}_{n}\right]}{[\mathrm{M}] \cdot[\mathrm{L}]^{n}}
$$

对式(1), 当 $[\mathrm{M}]=\left[\mathrm{ML}_{n}\right]$, 则:

$$
K_{\text {稳 }}=\frac{1}{[\mathrm{~L}]^{n}}
$$

假设配合物总浓度为 $c$, 由于配合物不稳定, 存在解离现象, 其解离度为 $\alpha$, 从而有:

$$
\begin{aligned}
& {\left[\mathrm{ML}_{n}\right]=(1-\alpha) \cdot c} \\
& {[\mathrm{M}]=\alpha \cdot c} \\
& {[\mathrm{~L}]=\alpha \cdot n \cdot c}
\end{aligned}
$$

代入式(1)有:

$$
K_{\text {稳 }}=\frac{1-\alpha}{n^{n} \cdot \alpha^{n+1} \cdot c^{n}}
$$

假设配合物络合比 $n=1$, 则式(3)可简化为:

$$
K_{\text {稳 }}=\frac{1-\alpha}{\alpha^{2} \cdot c}
$$

根据式(2), 当 $\left[\mathrm{Cu}^{2+}\right]=[\mathrm{CuL}]$, 即有 $A=0.5 A_{\text {max }}$ 时, 作水平线与曲线交于 $B$ 点, $B$ 点对应横坐标为 $n_{B}$, 从而可求得 $0.5 A_{\max }$ 对应 $\mathrm{H}_{3} \mathrm{~L}$ 浓度 $c_{\mathrm{L}}$ 的值, 相关数据见表 2 。将 $c_{\mathrm{L}}$ 的值代入式(2)可求得配合物稳定

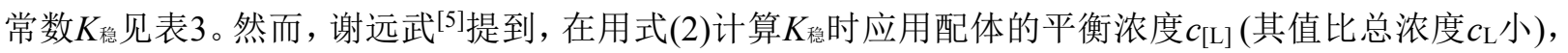
而表 3 是用总浓度 $c_{\mathrm{L}}$ 进行计算, 因此会有较大误差(见表 3 ); 因平衡浓度 $c_{[\mathrm{L}]}$ 难以根据现有条件求出,

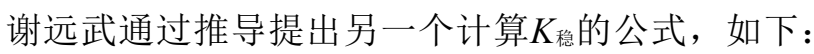

$$
K_{\text {稳 }}=\left(\frac{\varepsilon}{n}\right)^{n} \cdot \frac{A^{\prime}}{\left(A_{\max }-A^{\prime}\right)^{n+1}}
$$

式(5)中 $A^{\prime}$ 可由 $E$ 点引垂线与曲线的交点 $E^{\prime}$ 求得(图1), 而 $\varepsilon$ 为配合物的摩尔吸收系数, 可由实验数 据计算得到:

根据朗伯比尔定律 $A=\varepsilon b c$, 已知 $A_{\max }$ 为磺基水杨酸铜未解离时的吸光度, 从而有 $A=A_{\max }=0.606$, 光 程即比色典厚度为 $b=1 \mathrm{~cm}$, 磺基水杨酸铜溶液浓度 $c=\frac{c_{\mathrm{Cu}\left(\mathrm{NO}_{3}\right)_{2}} \cdot V_{\mathrm{Cu}\left(\mathrm{NO}_{3}\right)_{2}}}{V_{\text {总 }}}=\frac{0.05000 \times 10.00}{50.00} \mathrm{~mol} \cdot \mathrm{L}^{-1}=$ $0.01000 \mathrm{~mol} \cdot \mathrm{L}^{-1}$, 从而得到磺基水杨酸铜的摩尔吸收系数 $\varepsilon=59.5$ 。

由图1知磺基水杨酸铜络合比为 1 , 结合之前的论述知, 可分别通过式(2)、(4)、(5)来求出其表现 
稳定常数 $K$ 稳, 相关结果见表 3 。然而, $K$ 稳是和实验条件密切相关的, 不能直接反映实验结果好坏, 要判断此数值是否合理需要进一步计算出绝对稳定常数 $K$, 并与理论值进行比较。

表3 通过不同公式计算稳定常数的差别

\begin{tabular}{ccccc}
\hline 稳定常数计算公式 & $K_{\text {稳 }}$ & $\lg K_{\text {稳 }}$ & $\lg \alpha_{\mathrm{L}(\mathrm{H})}$ & $\lg K$ \\
\hline$(2)$ & 164 & 2.21 & 6.60 & 8.81 \\
$(4)$ & 1075 & 3.03 & 6.60 & 9.63 \\
$(5)$ & 1052 & 3.02 & 6.60 & 9.62 \\
\hline
\end{tabular}

$\alpha_{\mathrm{L}(\mathrm{H})}$ 为 $\mathrm{H}_{3} \mathrm{~L}$ 的酸效应系数, $K$ 为绝对稳定常数, 相关计算公式见式(6)、(7); 根据文献 ${ }^{[6]}$, 绝对稳定常数理论值为 $\lg K_{\mathrm{CuL}}=9.52$ 。

相关计算公式如下:

$$
\begin{aligned}
& \alpha_{\mathrm{L}(\mathrm{H})}=1+\frac{\left[\mathrm{H}^{+}\right]}{K_{\mathrm{a} 3}}+\frac{\left[\mathrm{H}^{+}\right]^{2}}{K_{\mathrm{a} 3} \cdot K_{\mathrm{a} 2}} \\
& \lg K=\lg K_{\text {稳 }}+\lg \alpha_{\mathrm{L}(\mathrm{H})}
\end{aligned}
$$

$\left[\mathrm{H}^{+}\right]$为溶液氢离子浓度; $\mathrm{H}_{3} \mathrm{~L}$ 第一步是全部离解的, $K_{\mathrm{a} 2}$ 和 $K_{\mathrm{a} 3}$ 分别是其第二和第三级离解常数, 根据文献 ${ }^{[7]}$ 知 $K_{\mathrm{a} 3}=10^{-11.6}, K_{\mathrm{a} 2}=10^{-2.6}$ 。

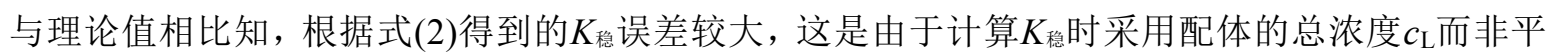
衡浓度 $c_{[\mathrm{L}]}$ 所导致。根据式(4)、式(5)所得结果一致, 也与理论值较为接近, 表明摩尔比法可用这两个 公式来计算 $K$ 稳。

\section{3 磺基水杨酸铜溶液定容前后 $\mathrm{pH}$ 差别与稳定常数的计算}

由式(6)、式(7)可知, 通过实验数据计算得到的 $\mathrm{CuL}$ 绝对稳定常数大小与溶液 $\mathrm{pH}$ 密切相关。实验 中发现尽管溶液已调节到指定 $\mathrm{pH}$ (例如 4.70), 定容所用的硝酸钾也是同样的 $\mathrm{pH}$, 但在定容后, 溶 液的 $\mathrm{pH}$ 会发生升高的现象(例如7号溶液定容前是 4.70, 定容后测得 5.00)。这是由于配合物溶液定容 前后浓度变化导致其解离度也发生了变化, 从而影响了溶液 $\mathrm{pH}$ 。由图 1 可知, 稳定常数的计算所用数 据是以 $E^{\prime}$ 数据为基础的, $E^{\prime}$ 在图上很接近 7 号溶液对应的点, 在此以 7 号溶液为例阐述配合物溶液 $\mathrm{pH}$ 在定容前后发生变化的原因:

$\mathrm{H}_{3} \mathrm{~L}$ 是一个三元酸, 但它的第三级解离常数很小 $\left(K_{\mathrm{a} 3}=10^{-11.6}\right.$, 用 $\mathrm{NaOH}$ 标准溶液滴定时, 只能与 前两级解离的 $\mathrm{H}^{+}$反应形成一个突跃) ${ }^{[8]}$, 因此, 它与铜离子在一定 $\mathrm{pH}$ 下发生反应如下:

$$
\begin{aligned}
& \mathrm{H}_{3} \mathrm{~L}+2 \mathrm{OH}^{-} \rightarrow \mathrm{HL}^{2-}+2 \mathrm{H}_{2} \mathrm{O} \\
& \mathrm{HL}^{2-}+\mathrm{Cu}^{2+} \rightleftharpoons \mathrm{CuL}^{-}+\mathrm{H}^{+}
\end{aligned}
$$

在调节 7 号溶液 $\mathrm{pH}$ 到 4.70 后, 烧杯中溶液总体积为 $12 \mathrm{~mL}$ 左右, 此时 $\mathrm{CuL}$ 浓度为 $c_{0}$, 解离度为 $\alpha_{0}$; 将溶液定容于 $50 \mathrm{~mL}$ 容量瓶后, $\mathrm{CuL}$ 浓度被稀释为 $0.25 c_{0}$, 此时解离度变为 $\alpha_{1}$ 。CuL浓度在定容后变小 了, 导致 $\alpha_{1}>\alpha_{0}$, 也就是定容后有更多的 $\mathrm{CuL}$ 发生了式(9)的逆反应, 消耗了溶液中的部分氢离子, 从 而使得溶液 $\mathrm{pH}$ 从 4.70 上升为 5.00 。 $\mathrm{pH}$ 不同, 计算得到的绝对稳定常数也有差别。从表 4 的 $\lg K$ 值与理 论值9.52相比知, 在计算绝对稳定常数的时候, 应采用定容后的 $\mathrm{pH}$ 。

表4 通过定容前后pH计算绝对稳定常数的差别

\begin{tabular}{ccccc}
\hline 测量点 & $\mathrm{pH}$ & $\lg \alpha_{\mathrm{L}(\mathrm{H})}$ & $\lg K_{\text {稳 }}$ & $\lg K$ \\
\hline 定容前 & 4.70 & 6.90 & 3.03 & 9.93 \\
定容后 & 5.00 & 6.60 & 3.03 & 9.63 \\
\hline
\end{tabular}




\section{3 结语}

本文采用摩尔比法测定磺基水杨酸铜组成与稳定常数。通过实验发现, 在铜离子浓度一定的前 提下, 5-磺基水杨酸浓度大于等于铜离子浓度四倍时, 配合物溶液的吸光度达到最大值并基本维持 不变。实验还发现配制系列配合物溶液过程中, 由于定容前后体积发生变化, 其解离度也发生变化, 从而导致定容前后的配合物溶液 $\mathrm{pH}$ 不同, 在计算绝对稳定常数时, 应采用定容后的 $\mathrm{pH}$ 。

实验通过摩尔比法测得磺基水杨酸铜在 $\mathrm{pH}$ 为 5 时络合比为 1 , 然而其表现稳定常数 $K$ 稳不能通过 式(2)计算, 可由式(4)或式(5)求得。由式(4)求得其绝对稳定常数 $\lg K=9.63$, 与理论值9.52接近, 表明 本文设计的摩尔比法测定磺基水杨酸铜组成与稳定常数的实验方案可行。

\section{参 考 文 献}

[1] 郭峰, 冯武, 朱元成. 天水师范学院学报, 2003, 23 (2), 37.

[2] 石建新, 巢晖. 无机化学实验. 第4版. 北京: 高等教育出版社, 2019: 120.

[3] 高吉刚, 周杰, 盛锋. 分析化学研究简报, 2002, 30 (5), 594.

[4] 张淑艳, 阿其夫. 内蒙古民族大学学报(自然科学版), 2002, 17 (2), 114.

[5] 谢远武. 化学通报, 1988, No. 2, 39 .

[6] Speight, J. G. Lange's Handbook of Chemistry, 16th ed.; McGraw-Hill: New York, NY, USA, 2005; p. 8.102.

[7] 武汉大学. 分析化学. 第6版. 北京: 高等教育出版社, 2016: 219.

[8] 王鸿显, 马淮凌, 李贯良. 黄淮学刊, 1992, 8 (2), 68. 